\title{
The species of Alangium section Rhytidandra (Alangiaceae)
}

\author{
W.J.J.O. de Wilde ${ }^{1}$, B.E.E. Duyfjes ${ }^{1}$
}

Key words

Alangiaceae

Alangium sect. Rhytidandra

Australia

new species

Pacific

SE Asia

taxonomy

\begin{abstract}
Alangium sect. Rhytidandra is confined to SE Asia, the Pacific and East Australia, and contains 13 species. Five species, viz. Alangium brassii, A. glabrum, A. gracile, A. guadalcanalense, and A. velutinum are described as new, whereas $A$. villosum subsp. solomonense is raised to specific rank: $A$. solomonense. Subspecies tomentosum formerly in A. villosum is transferred to another species and has become A. polyosmoides subsp. tomentosum. Finally lodes ferruginea, described in Icacinaceae appeared to belong in Alangiaceae, and as the name ferruginea was occupied in Alangium it is changed into A. strigosum, nom. nov. A key to the species is presented, and the concerned taxa are enumerated, referenced, described, and two are figured.
\end{abstract}

Published on 20 April 2017

\section{INTRODUCTION}

There are four sections in the genus Alangium and the number of species occurring in Malesia is estimated at c. 38 (but there are some collections which cannot be placed, and wait for additional material to be described). The sections are: sect. Alangium (see De Wilde \& Duyfjes in press) with four species in Malesia, sect. Conostigma (see De Wilde \& Duyfjes 2017) with 19 species in Malesia, sect. Marlea (Roxb.) Baill. with five species in Malesia, and according to the present revision, sect. Rhytidandra with 10 species in Malesia.

Alangium sect. Rhytidandra was established by Bloembergen (1939). The section comprises the group of species with stamens equal in number to petals and is furthermore defined by a deeply 2-lobed stigma and petals generally more or less connate at base. The section is distributed from Java eastwards into East Malesia, the Pacific and East Australia.

After deliberate consideration Bloembergen (1939: 201) decided to accept for the section only one single species, Alangium villosum Blume, with ten geographically separated subspecies and an eleventh undescribed subspecies of which at the time only sterile material was at hand. The present authors reconsidered the matter and, with much more material available than in 1939, accept thirteen species with one including two subspecies. Five species are here described as new, whereas one species needed a new name, $A$. strigosum (for lodes ferruginea). Some of Bloembergen's subspecies are re-instated as species and one is raised to specific rank: Alangium solomonense. The above-mentioned 11th subspecies seen by Bloembergen (1939: 210) appeared, with the aid of subsequently collected fertile material, to belong to sect. Conostigma Bloemb. (see De Wilde \& Duyfjes 2017) and has been recognised as a new variety of Alangium maliliense Bloemb.

The mode of venation, used as a character for species distinction, viz. lamina at base palminerved (or 3-plinerved) vs lamina entirely pinninerved is not always very clear. Leaves with weak basal veins may pass for entirely pinninerved.
The species of sect. Rhytidandra generally have domatia in (part of) the axils of the lateral nerves, or sometimes just glands close to the nerve axils. They seem to be absent in, for example, A. guadalcanalense and $A$. villosum. Some species in sect. Alangium also have domatia.

The basically cymose inflorescences in sect. Rhytidandra are here described as 'simple' or 'compound'. It denotes, as far as the limited material seen allows, a distinction which may be helpful in the definition of species. In a 'compound' cyme the peduncle continues above the (sub)opposite first lateral branches not with a single flower but reiterates in a more complex several-flowered central branch. This situation is prevalent also in, for example, A. griffithii of sect. Marlea.

In the present paper, all species of Alangium sect. Rhytidandra are enumerated, referenced and briefly annotated where appropriate.

Alangium Lam. sect. Rhytidandra (A.Gray) Baill.

Alangium Lam. sect. Rhytidandra (A.Gray) Baill. (1864-1865) 193; (1876) 270; Bloemb. (1939) 197. - Rhytidandra A.Gray (1854) 302, t. 28. - Type: Rhytidandra vitiense A.Gray (= Alangium vitiense (A.Gray) Baill.).

Shrubs or trees. Inflorescences a single peduncled cyme. Flowers: petals free or connate at base; stamens as many as petals; filament longer or shorter than anther; stigma 2-armed, stigmatic on the inside. Fruit several per infructescence, ellipsoid or ovoid, more or less flattened. Endosperm smooth, radicle shorter than half the length of the cotyledons.

Distribution - Java east into the Pacific, Fiji, New Caledonia, E Australia.

Thirteen species are recognised.

\footnotetext{
Naturalis Biodiversity Center, section Botany, P.O. Box 9517, 2300 RA Leiden, The Netherlands;

corresponding author e-mail: b.dewilde-duyfjes@naturalis.nl.
} 


\section{KEY TO THE SPECIES OF}

\section{ALANGIUM SECTION RHYTIDANDRA}

The key presented here is largely based on flowering specimens. Although construction of a dichotomous key is impractical for fruiting specimens, most can nevertheless be identified with the help of fruit size and provenance, as follows:

\begin{tabular}{|c|c|}
\hline Fruit length $(\mathrm{mm})$ & species \\
\hline $8-12$ & pilosum (Philippines, Lesser Sunda Is.) \\
\hline $10-15$ & villosum (Java) \\
\hline $12-15$ & $\begin{array}{l}\text { strigosum (Papua New Guinea: Madang, } \\
\text { Sepik) }\end{array}$ \\
\hline $13-15$ & brassii (Papua New Guinea: Milne Bay) \\
\hline 15 & $\begin{array}{l}\text { polyosmoides (E Australia), } \\
\text { velutinum (Papua New Guinea) }\end{array}$ \\
\hline $15-20$ & gracile (Papua New Guinea) \\
\hline $15-22$ & warburgianum (Moluccas: Bacan) \\
\hline $17-22$ & $\begin{array}{l}\text { solomonense (Solomon Is., Bougainville) } \\
\text { vitiense (Fiji, Vanuatu, New Caledonia) }\end{array}$ \\
\hline$(25-) 30$ & glabrum (Papua New Guinea) \\
\hline $30-35$ & ferrugineum (Moluccas, New Guinea) \\
\hline unknown & guadalcanalense (Solomon Is.) \\
\hline
\end{tabular}

1. Flowers larger; corolla in bud $9 \mathrm{~mm}$ long or more . . . . . 2

1. Flowers smaller; corolla in bud $8 \mathrm{~mm}$ long or less . . 10

2. Calyx lobes long-triangular, c. $1.5 \mathrm{~mm}$ long. - Bacan Isl. $\ldots \ldots \ldots \ldots \ldots \ldots \ldots \ldots$. . A. warburgianum

2. Calyx lobes \pm triangular, c. $0.5 \mathrm{~mm}$ long or lobes almost absent .......................

3. Ovary and calyx in bud reddish brown-hairy. Leaves drying reddish brown. - New Guinea . . . . . . . . . . . 4

3. Ovary and calyx in bud greyish brown or yellowish brownhairy. Leaves drying greenish. - New Guinea, Australia, Solomon Isl. . . . . . . . . . . . . . . . . 6

4. Corolla in bud slender, c. $1 \mathrm{~mm}$ diam. Peduncle c. $0.5 \mathrm{~mm}$ diam. Style glabrous. Fruit $15-20 \mathrm{~mm}$ long 4. A. gracile

4. Corolla in bud thickish, c. $1.5 \mathrm{~mm}$ diam. Peduncle $1-2 \mathrm{~mm}$ diam. Style (sparingly) hairy or glabrous. Fruit $15-35 \mathrm{~mm}$ long .................... 5

5. Lower lamina surface densely hairy but not velutinous. Style (sparingly) hairy. Fruit longly narrowed towards apex, 30-35 mm long. - Lowland forest . . 2 2. A. ferrugineum

5. Lower lamina surface velutinous. Style glabrous. Fruit not longly narrowed towards apex, c. $15 \mathrm{~mm}$ long. - Montane forest, $1400-2600 \mathrm{~m}$ altitude . . . . . 10. A. velutinum

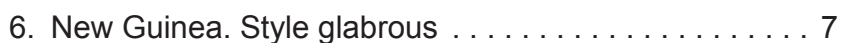

6. Australia or Solomon Isl. Style hairy . . . . . . . 9

7. Calyx lobes shorter than limb, in fruit hardly visible (rim of calyx almost straight), and not connivent ...1. A. brassii

7. Calyx lobes about as long as or longer than limb, in fruit connivent. . . . . . . . . . . . 8

8. Twig towards apex brown-hairy, hairs $0.5(-1) \mathrm{mm}$ long. Fruit c. $15 \mathrm{~mm}$ long . . . . . . . . . . 9. A. strigosum

8. Twig glabrescent (hairs less than $0.5 \mathrm{~mm}$ long). Fruit (25-)30 mm long . . . . . . . . . . . . . 3. A. glabrum

9. Filaments completely fused with the tube of the corolla. Solomon Isl. (Guadalcanal Isl.) . . 5. A. guadalcanalense

9. Filaments largely free. - East Australia . . . . . . . . . .

$$
\text { 7. A. polyosmoides (2 subsp.) }
$$

10. Corolla in bud 4-6(-7) mm long. - Philippines, Lesser Sunda Isl. (Moluccas) . . . . . . . . . . . . 6. A. pilosum

10. Corolla in bud $(6-) 7-8 \mathrm{~mm}$ long . . . . . . . . 11

11. Twig towards apex, leaves, inflorescences and flowers densely hairy. - Java .............11. A. villosum
11. Twig towards apex, leaves, inflorescences and flowers (sub)glabrous. - Pacific area . . . . . . . . . 12

12. Style glabrous or sparingly hairy. - Fiji, Vanuatu, New Caledonia ................... . vitiense

12. Style hairy. - Solomon Isl. . . . . . 8. A. solomonense

\section{Alangium brassii W.J.de Wilde \& Duyfjes, sp. nov.}

Resembling Alangium glabrum W.J.de Wilde \& Duyfjes but differing in its conspicuous golden or greyish brown indument and small fruit c. $15 \mathrm{~mm}$ long (whole plant glabrescent and fruit c. $30 \mathrm{~mm}$ long in A. glabrum). - Type: Brass 22578 (holo L L2498023), Papua New Guinea, Milne Bay Province, North slopes of Mt Dayman, Maneau Range, 2000 m alt., 27 May 1953, fl., fr.

Etymology. The species is named after its collector L.J. Brass.

Slender treelet 1-5 m tall; twigs 1.5-2.5(-4) mm diam, dark brown, at apex and leaf bud(s) with (dense) golden or greyish brown hairs $0.1-0.3 \mathrm{~mm}$ long. Leaves: petiole $0.4-0.6 \mathrm{~cm}$ long, late glabrescent; lamina subcoriaceous, drying greenish, glabrescent (except midrib at base below), elliptic, $7-12$ by $2.5-5$ $\mathrm{cm}$, base asymmetric, attenuate, apex acute-acuminate; veins 4 or 5 at each side, tertiary venation reticulate, ultimate areoles small, distinct. Inflorescences hairy as the twigs, glabrescent, simple, 2-5-flowered, peduncle 5-10 mm long. Flowers minutely (pale) greyish brown-hairy, hairs c. $0.1 \mathrm{~mm}$ long; pedicel 2-3 mm long; corolla in bud 10-12 mm long, c. $1.5 \mathrm{~mm}$ diam, base not swollen, apex obtuse; ovary and calyx c. $2.5 \mathrm{~mm}$ long, not ribbed, greyish or yellowish brown-hairy, limb c. $1 \mathrm{~mm}$ long, at margin c. $2 \mathrm{~mm}$ wide, unlobed (straight) or faintly lobed, not spreading; petals 4, inside glabrous, except minutely hairy at base, connate into a tube $0.5-1 \mathrm{~mm}$ long; stamens 4 , filament c. $5 \mathrm{~mm}$ long, minutely hairy, anther c. $5 \mathrm{~mm}$ long, the thecae slightly sagittate at base, connective glabrous; style glabrous. Fruit 1-3 per infructescence, ripening colour unknown, glabrous, obovoid-ellipsoid, $13-15$ by $6-7 \mathrm{~mm}$, finely $8-10$-ribbed, base \pm narrowed, apex nearly rounded; calyx remnant minute.

Distribution - Papua New Guinea (Milne Bay Prov., North slopes of Mt Dayman).

Habitat \& Ecology - Abundant in undergrowth in dryish forest of slopes, occasional in mossy forest; 2000-2230 m altitude; flowering and fruiting in May and June.

Field-note - Flowers yellow.

Notes - 1. Alangium brassii is known from two collections, both from about the same locality, viz. the type, Brass 22578 'abundant in undergrowth, dryish forest of slopes' at $2000 \mathrm{~m}$ alt. and Brass $22698(\mathrm{~L})$ 'occasional in mossy forest undergrowth' at $2230 \mathrm{~m}$ alt.

2. As common in species of sect. Rhytidandra domatia are present in the axils of the lateral veins. In A. brassii, however, there are no real domatia but rather distinct round glands at some distance from the vein axils.

\section{Alangium ferrugineum C.T.White - Fig. 1}

Alangium ferrugineum C.T.White (1929) 248; Bloemb. (1935) 272, f. 3g, h. - Alangium villosum (Blume) Wangerin subsp. ferrugineum (C.T.White) Bloemb. (1939) 206, f. 6f, 7m, n. - Type: Brass 1066 (holo BRI BRI AQ0382983; iso L L0009829, P P04552634), Papua New Guinea, Gulf Province, Aroara, Vailala River, 60 m alt., 25 Feb. 1926.

Shrub or tree 5-10 m tall; twigs brown, 2-3 mm diam, yellowish, ferruginous, or rusty hairy, hairs c. $0.5 \mathrm{~mm}$ long. Leaves: petiole $0.5-1.5 \mathrm{~cm}$ long, hairy; lamina drying reddish brown, densely hairy, (ovate-)elliptic, $11-18$ by $5-8.5 \mathrm{~cm}$, base asymmetric, rounded or (short) cuneate, apex acute-acuminate; veins pinnate, $5-8$ at each side, tertiary venation thin, (sub)scalariform. Inflorescences hairy as the twigs, simple, 2-8-flowered, peduncle 5-15 mm long, 1-1.5 mm diam. Flowers densely hairy; pedicel c. $3 \mathrm{~mm}$ long; corolla in bud c. $12 \mathrm{~mm}$ long (c. $15 \mathrm{~mm}$ 


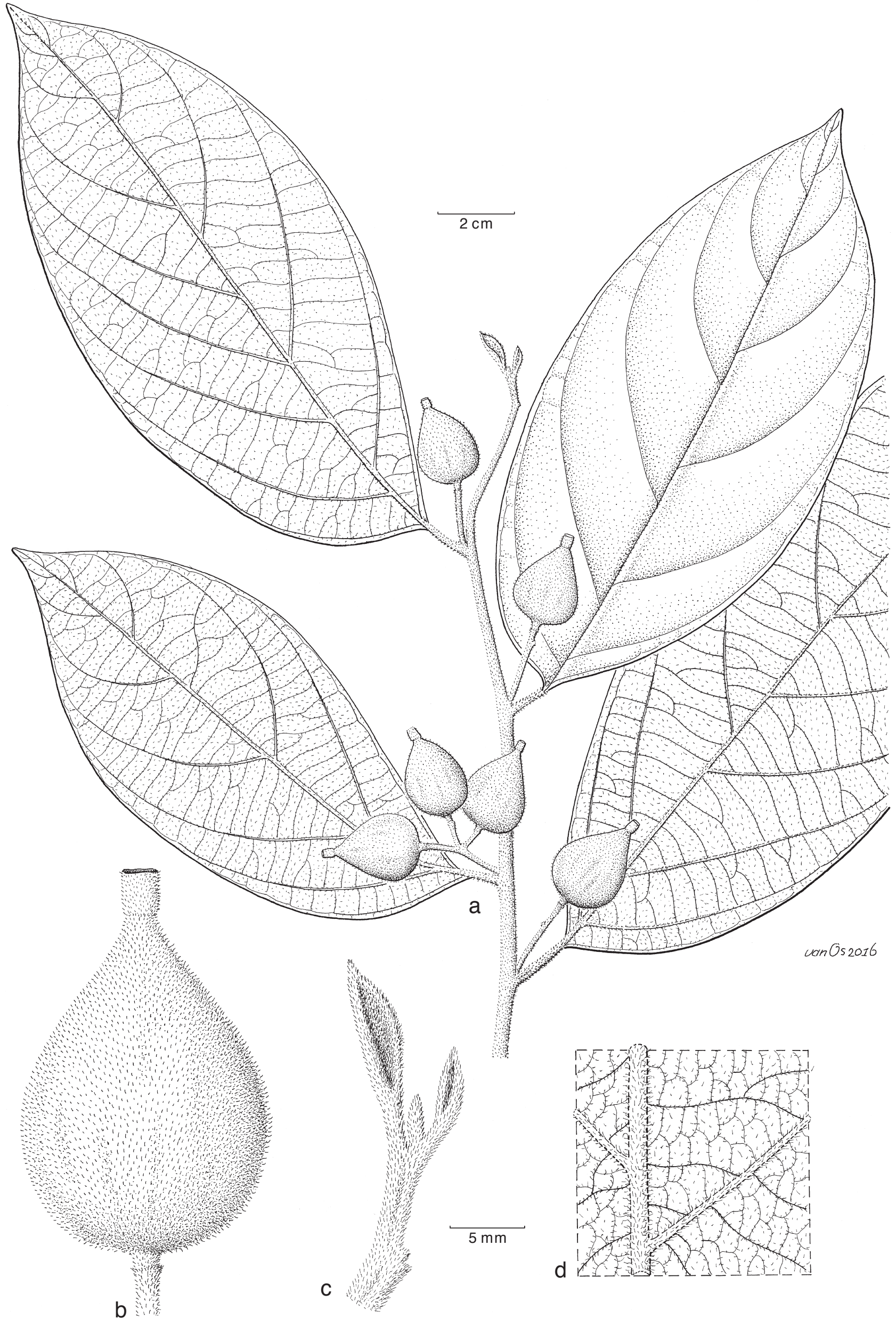

Fig. 1 Alangium ferrugineum C.T.White. a. Fruiting twig; b. fruit; c. twig apex; d. detail of lower lamina surface (all: Nooteboom 5773, L). - Drawn by Jan van Os. 
long when fresh), c. $1.5 \mathrm{~mm}$ diam, base not swollen, apex subobtuse; ovary and calyx c. $2.5 \mathrm{~mm}$ long, not constricted below the limb, not ribbed, reddish brown-hairy; limb c. $1 \mathrm{~mm}$ long, not spreading, with 5 obtuse lobes c. $0.5 \mathrm{~mm}$ long; petals 5 , inside glabrous, c. $12 \mathrm{~mm}$ long, connate at base into a tube c. $2 \mathrm{~mm}$ long; stamens 5, filament 3-5 mm long, hairy, anther $8(-10) \mathrm{mm}$ long, connective glabrous; style 6-10 mm long, (sparingly) hairy, stigma lobes curved, c. $3 \mathrm{~mm}$ long. Fruit 1 or 2 (or 3?) per infructescence, ripening yellow (?), densely brown-hairy, ovoid and longly narrowed towards apex, 30-35 $\mathrm{mm}$ long, (10-)15-20 mm broad, not ribbed; calyx remnant small, narrow, subtruncate.

Distribution - Moluccas (Aru Isl. (Pulau Kobroor)), Papua; Papua New Guinea (Madang \& Gulf Provinces).

Habitat \& Ecology - Alluvial forest, foothill forest, secondary forest, clayey soil, coral stone; sea level to $400 \mathrm{~m}$ altitude; flowering in February and August; fruiting in February and July to November.

Field-notes - Handsome tree; flowers white; fruit yellow. Vernacular name - Ala po'u (Aru IsI.).

Additional specimens studied. Moluccas, Nooteboom 5773, Aru Isl. (P Kobroor), 0-50 m alt., 27 Apr. 1993, fr. - PAPUA, Aet (exp. Lundquist) 558, South New Guinea, Patawai near Oeta, 21 July 1941, fr.; Versteegh BW 4850, near Wage, Div. South New Guinea, along River Digoel, $5 \mathrm{~m}$ alt., 16 Sept. 1957, fr. - Papua New GuineA, Madang Prov., Josephstaal area, Takeuchi et al. 13103, $424 \mathrm{~m}$ alt., fr.; Takeuchi et al. 13674, $160 \mathrm{~m}$ alt., 1 Dec. 1999, fr.; Takeuchi et al. 13828, 80 m alt., 9 Aug. 1999, fl.; Brass 1066 (type), Gulf Prov., Aroara, Vailala River, 60 m alt., 25 Feb. 1926.

\section{Alangium glabrum W.J.de Wilde \& Duyfjes, sp. nov.}

Resembling Alangium strigosum W.J.de Wilde \& Duyfjes but differing in whole plant glabrescent from minute hairs $0.1 \mathrm{~mm}$ long, lamina $8-11 \mathrm{~cm}$ long, and fruit c. $30 \mathrm{~mm}$ long (twigs densely rusty or golden brown-hairy, hairs 0.5-1 mm long, lamina $13-19 \mathrm{~cm}$ long, and fruit $12-15 \mathrm{~mm}$ long in A. strigosum). - Type: Womersley \& Woolliams NGF 37092 (holo L L2500143; iso A, BO, BRI, CANB, K, LAE, SING, SYD, all n.v.), Papua New Guinea, Southern Highlands Province, Sawmill at lalibu, 2000 m alt., 14 Sept. 1967, fl., fr.

Etymology. The specific epithet 'glabrum' refers to the overall glabrous aspect of the plant.

Tree c. $12 \mathrm{~m}$ tall; twigs brown, 1-2.5 mm diam, whole plant glabrescent from minute greyish brown hairs c. $0.1 \mathrm{~mm}$ long. Leaves: petiole $0.6-0.8 \mathrm{~cm}$ long; lamina drying brownish green, elliptic, $8-11$ by $3.5-5 \mathrm{~cm}$, base (slightly) asymmetric, rounded or broadly short-cuneate, apex long acute-acuminate; veins ( 3 or) 4 or 5 at each side, tertiary venation reticulate. Inflorescences minutely hairy as the twigs, glabrescent, simple, 2- or 3-flowered, peduncle (5-)10 mm long. Flowers densely minutely brown-hairy, hairs c. $0.1 \mathrm{~mm}$ long; pedicel c. $2 \mathrm{~mm}$ long; corolla in bud (only seen in immature flowers) presumably $8-10 \mathrm{~mm}$ long, 1(-1.5) $\mathrm{mm}$ diam, base not swollen, apex narrowly obtuse; ovary and calyx c. $3.5 \mathrm{~mm}$ long, coarsely 4-8-ribbed, minutely greyish brown-hairy; limb c. $1.5 \mathrm{~mm}$ long, including 4 triangular lobes $0.7-1 \mathrm{~mm}$ long, somewhat spreading; petals 4 , inside thinly hairy towards base; stamens 4, mature filaments and anther not known; immature filament sparingly hairy, connective glabrous; style glabrous. Fruit 1-3 per infructescence, ripening green (?), glabrescent (except at very apex), narrowly ellipsoid, broadest slightly below the middle and pointed (narrowed) at both ends, (25-) $30 \mathrm{~mm}$ long, 10-11 mm diam, (when dry) shallowly 4- (or 8-)grooved and irregularly dimpled; calyx remnant small, narrow, with the 4 calyx lobes connivent.

Distribution - The species is only known from the type.

Habitat \& Ecology - In Nothofagus dominated rain forest; c. $2000 \mathrm{~m}$ altitude; flowering and fruiting in September.

Field-note - Fruit green.

Note - The fruiting pedicel is remarkably long, $5-7 \mathrm{~mm}$.

\section{Alangium gracile W.J.de Wilde \& Duyfjes, sp. nov.}

Resembling Alangium ferrugineum C.T.White but differing in its slender and long peduncle, $8-40 \mathrm{~mm}$ long, c. $0.5 \mathrm{~mm}$ diam, and fruit glabrescent, 15-20 $\mathrm{mm}$ long (peduncle 5-15 mm long, 1-1.5 mm diam, and fruit densely hairy, 30-35 mm long in A. ferrugineum). - Type: Forman et al. LAE 52499 (holo L L2500089; iso A, BRI, CANB, K, LAE, all n.v.), Papua New Guinea, ridge SW of Efogi Village, Central Province, Port Moresby Subdistrict, S9 $10^{\prime}$ E147 $39^{\prime}, 1370$ m alt., 25 Sept. 1973, fl.

Etymology. The species epithet 'gracile' refers to its slender peduncle and corolla in bud.

Shrub or tree 3-7(-15) m tall; twigs (dark) brown, 1.5-3(-5) $\mathrm{mm}$ diam, ferruginous or rufous-hairy, hairs $0.2-0.5 \mathrm{~mm}$ long. Leaves: petiole $0.5-1 \mathrm{~cm}$ long, hairy; lamina drying reddish brown, hairy or late glabrescent, elliptic, $7-15(-17)$ by $3-6(-8)$ $\mathrm{cm}$, base hardly asymmetric, cuneate, apex acute-acuminate; veins pinnate, $5-8$ at each side, tertiary venation (reticulate and) scalariform. Inflorescences hairy as the twigs, simple, 3-10-flowered, peduncle slender, $8-40 \mathrm{~mm}$ long, c. $0.5 \mathrm{~mm}$ diam. Flowers densely hairy, hairs rufous, $0.3-0.5 \mathrm{~mm}$ long; pedicel 1-3 mm long; corolla in bud c. $10 \mathrm{~mm}$ long, slender, c. $1 \mathrm{~mm}$ diam, base not swollen, apex subacute; ovary and calyx densely coarsely rufous-hairy, c. $2.5 \mathrm{~mm}$ long, constricted below the limb, not ribbed; limb $0.5-1 \mathrm{~mm}$ long, spreading, with 5 low, obtuse lobes less than $0.5 \mathrm{~mm}$ long; petals 5 , inside glabrous (or hairs extremely minute), c. $10 \mathrm{~mm}$ long, connate at base into a tube 1-1.5 mm long; stamens 5, c. $10 \mathrm{~mm}$ long, filament c. $6 \mathrm{~mm}$ long, anther 3(-4) mm long, connective glabrous; style c. $8 \mathrm{~mm}$ long, glabrous, stigma lobes c. $1 \mathrm{~mm}$ long. Fruit $1(-3)$ per infructescence, ellipsoid-fusiform, 15-20 mm long, early glabrescent, smooth or finely 5-ribbed; calyx remnant small, spreading, like calyx limb in flower, hairy or late glabrescent.

Distribution - Papua New Guinea (Eastern Highlands and Central Provinces).

Habitat \& Ecology - Montane forest, stream side, scattered in forest; 1060-2600 m altitude; flowering from September to December; fruiting from September to November.

Field-notes - Fruit ochre suffused red; fruit red.

Additional specimens studied. Papua New Guinea, Central Prov., Boridi, Carr 13273, 1525 m alt., 20 Sept. 1935, fr.; Carr 14214, 1460 m alt., 27 Sept. 1935, fl.; Carr 14486, 1370 m alt., 9 Oct. 1935, fr.; Carr 14684, 1066 m alt., 22 Oct. 1935, fl.; Foreman \& Vinas LAE 60189, S905' E147³8', 1370 m alt., 30 Sept. 1973, fl.; Central Prov., Lala River (most likely Laila River), Carr 14095, 1830 m alt., 30 Dec. 1935, fl.; Central Prov., Efogi, Croft \& Vinas NGF 34867,1400 m alt., 21 Sept. 1973, fr.; Forman et al. LAE 52499 (type), S9 $10^{\prime} \mathrm{E} 147^{\circ} 39^{\prime}, 1370$ m alt., 25 Sept. 1973, fl.; Stevens LAE 50424, S9¹0' E147 $35^{\circ}, 1110$ m alt., 5 Sept. 1970, fl., fr.; Eastern Highlands Prov., Stevens LAE 50498, Marafunga, $\mathrm{S}^{\circ} 05^{\prime} \mathrm{E} 145^{\circ} 15^{\prime}, 2600 \mathrm{~m}$ alt., fl., fr.

Notes -1 . Alangium gracile is vegetatively similar to $A$. ferrugineum, but differs in having inflorescence and flowers more coarsely hairy, peduncle more slender, and fruit smaller; ovary and calyx limb differ also considerably, not constricted below the limb in $A$. ferrugineum. In $A$. gracile ovary and limb are reminiscent of those of $A$. pilosum, but these are much less conspicuously hairy.

2. Like in A. ferrugineum, also in A. gracile the leaves have the lateral veins frequently tending to be 3-plinerved at base.

\section{Alangium guadalcanalense W.J.de Wilde \& Duyfjes, sp. nov.}

Resembling Alangium polyosmoides (F.Muell) Baill. subsp. tomentosum (F.Muell) W.J.de Wilde \& Duyfjes but differing in its filaments completely fused with the $3 \mathrm{~mm}$ long basal corolla tube (base of filaments slightly adnate to petals in A. polyosmoides subsp. tomentosum). - Type: Gafui \& collectors BSIP (holo L L.2183210; iso BSIP n.v.), Solomon Isl., West coast of Guadalcanal Isl., Kambi Hill, 600 m alt., 14 June 1968, fl.

Etymology. The specific epithet 'guadalcanalense' refers to the collection locality, Guadalcanal IsI. 
Tree c. $6 \mathrm{~m}$ tall; twigs greyish brown, 2-4 mm diam, densely greyish brown-hairy, hairs $0.5(-1) \mathrm{mm}$ long. Leaves: petiole $0.5-0.7 \mathrm{~cm}$ long; lamina drying brownish green, hairy at both sides (upper side partly late glabrescent), ovate(-elliptic), 10-12 by $4.5-6.5 \mathrm{~cm}$, base strongly asymmetric, rounded to short acuminate, apex acute-acuminate; veins $5-7$ at each side, tertiary venation reticulate or faintly scalariform, domatia absent. Inflorescences densely hairy, solitary peduncled (compound) cymes, 4-7-flowered; peduncle 6-7 mm long. Flowers densely hairy as the twigs; pedicel $1-2 \mathrm{~mm}$ long; corolla in bud $9-10 \mathrm{~mm}$ long, base not swollen, apex narrowly obtuse; ovary and calyx 3-4 mm long, not ribbed, greyish brown-hairy; limb 1.5-2 mm long, not spreading, at margin $2-2.5 \mathrm{~mm}$ wide, with 5 small lobes; petals 5, inside hairy, $9-12 \mathrm{~mm}$ long, at base for 3-4 mm connate into a tube; stamens 5; filament (3-)4 mm long, completely adnate to the hairy tube of the corolla, anther c. $6 \mathrm{~mm}$ long, inserted in the calyx at apex of tube, connective glabrous; style 6-7 mm long, hairy, stigma lobes c. $1.5 \mathrm{~mm}$ long. Fruit not known.

Distribution - Solomon Isl. (Guadalcanal Isl.), known only from the type.

Habitat \& Ecology - Well drained primary forest, ridge top; c. $600 \mathrm{~m}$ altitude; flowering in June.

Field-notes - Bole straight but leaning; bark surface grey, smooth.

Note - In general appearance reminiscent of $A$. polyosmoides subsp. tomentosum from Australia but markedly different in the filaments completely fused with the corolla tube, rendering the thecae sessile in the tube.

\section{Alangium pilosum Merr. - Fig. 2}

Alangium pilosum Merr. (1922) 417; (1923) 241. - Alangium villosum (Blume) Wangerin subsp. pilosum (Merr.) Bloemb. (1939) 205, f. 6d, 7h-i. - Type: Ahern's collector FB 3307 (holo PNH†, lectotype here designated: US US00127564; iso K n.v.), Luzon, Rizal Prov., Sept. 1905.

Alangium pilosum Merr. var. subglabrum Merr. (1922) 418; (1923) 241. Type: Contreras FB 23399 (holo $\mathrm{PNH}$, lectotype here designated: US US00127565; iso K K000704822,), Negros, 300 m alt., 14 July 1914, fl.

Alangium villosum (Blume) Wangerin var. parviflorum Bloemb. (1935) 272. - Alangium villosum (Blume) Wangerin subsp. parviflorum (Bloemb.) Bloemb. (1939) 206. - Type: Boschproefstation bb 11370 (holo BO; iso L L0986670), Flores, Boehe Soge (Maoemere), 250 m alt., 7 Feb. 1927, fl.

Shrub or tree 4-20 m tall; twigs brown, 1.5-3(-4) mm diam, grey or pale brown-hairy, hairs $0.1-0.3 \mathrm{~mm}$ long. Leaves: petiole $0.5-1 \mathrm{~cm}$ long, minutely hairy; lamina drying (greenish) brown, minutely hairy mainly on veins below, narrowly or broadly elliptic, $6-13$ by $2-4.5(-5) \mathrm{cm}$, base (strongly) asymmetric, cuneate, apex acute-acuminate; veins pinnate, $4-7$ at each side, tertiary venation reticulate (or partly scalariform). Inflorescences hairy (hairs $0.1-0.3 \mathrm{~mm}$ long), a single peduncled compound cyme, (3-)5-20-flowered; peduncle slender, 10-25 mm long. Flowers (densely) hairy, hairs $0.1-0.2 \mathrm{~mm}$ long; pedicel 2-5 $\mathrm{mm}$ long; corolla in bud 4-6(-7) $\mathrm{mm}$ long, base not swollen, apex subobtuse; ovary and calyx 2(-2.5) mm long, not ribbed; limb narrowly cup-shaped, little spreading, 0.5(-1) $\mathrm{mm}$ long, c. $1.5 \mathrm{~mm}$ wide, margin subentire or with 5 short lobes; petals $(4-) 5(-7)$, inside glabrous, connate at base for c. $0.5 \mathrm{~mm}$, $5-7(-8) \mathrm{mm}$ long; stamens 5 , at base slightly connate to petals, filament c. $2.5 \mathrm{~mm}$ long, hairy, anther $4-5 \mathrm{~mm}$ long, connective glabrous; style c. $5 \mathrm{~mm}$ long, glabrous, stigma lobes 1-2 mm long. Fruit 1-8 per infructescence, yellowish green, ripening black, glabrous, broadly ovoid-ellipsoid, 8-12 mm long, smooth or faintly narrowly 5-10-ribbed; calyx remnant minute.

Distribution - Philippines (Luzon, Leyte, Negros, Samar); Lesser Sunda Isl. (Lombok, Sumbawa, Flores, Timor).

Habitat \& Ecology - (Primary) forest; 15-900 m altitude; flowering all year round but mostly in February; fruiting mostly in April and May.
Field-notes - Bark greyish; fruit green, ripe fruit black.

Notes - 1. Plants from the Philippines are generally more densely and longer hairy (hairs $0.2-0.3 \mathrm{~mm}$ long) as compared with those of Lesser Sunda Isl., with hairs c. $0.1 \mathrm{~mm}$ long or plant occasionally subglabrous.

2. Two collections in L, from N Moluccas, Halmahera, viz. De Vogel 4419 (fl.) \& 4452 (fr.) may represent a new species. They key out on $A$. pilosum, but they look quite different by having broader more densely hairy leaves. The specimens are stored under Alangium aff. pilosum. An eventual description should wait for additional fruiting material.

\section{Alangium polyosmoides (F.Muell.) Baill.}

Alangium polyosmoides (F.Muell.) Baill. (1864) 195; Hewson (1984) 11, f. 3. - Pseudalangium polyosmoides F.Muell. (1860) 84. - Rhytidandra polyosmoides (F.Muell.) F.Muell. (1864) 172. - Type: Beckler s.n. (holo MEL MEL607318), Australia, New South Wales, Clarence River, fl.

Tree 4-20 m tall; twigs (blackish) brown, 1.5-3 mm diam, glabrous or (conspicuously) hairy. Leaves: petiole $(0.5-) 1(-1.5) \mathrm{cm}$ long; lamina drying brownish green, glabrous or hairy, ovate or elliptic, $6-12$ by $2.5-4 \mathrm{~cm}$, base (strongly) asymmetric, (sub)rounded or cuneate, apex acute-acuminate; veins pinnate, 4-6 at each side, tertiary venation faint or distinct, (reticulate or) scalariform. Inflorescences solitary peduncled compound cymes, glabrous or hairy, 2-10-flowered; peduncle 5-15 mm long. Flowers variously appressed (grey-)hairy; pedicel 1.5-4 $\mathrm{mm}$ long; corolla in bud (8-)10-15 mm long, base not swollen, apex (sub)obtuse or rounded; ovary and calyx \pm narrow, 2.5-3 mm long, not ribbed, greyish brown-hairy; limb (0.5-)1 $\mathrm{mm}$ long, not spreading, $1.5-2 \mathrm{~mm}$ wide at margin, with 5 low lobes; petals (4-)5-8, inside hairy or sparingly hairy, 8-15 $\mathrm{mm}$ long, somewhat coherent at base; stamens 5, (8-)10-14 $\mathrm{mm}$ long, at base slightly adnate to petals, filament $5-9 \mathrm{~mm}$ long, hairy in the middle, anther $3-5 \mathrm{~mm}$ long, the thecae usually slightly sagittate at base, connective glabrous; style $5-8$ $\mathrm{mm}$ long, hairy, stigma lobes c. $1 \mathrm{~mm}$ long. Fruit 1-6(-8?) per infructescence, ripening glossy black (always?), early glabrescent, ovoid-ellipsoid, c. $15 \mathrm{~mm}$ long; calyx remnant small.

Distribution - East Australia (Queensland to New South Wales).

Note - Alangium polyosmoides is a variable species, in which two taxa, apparently with some intermediates, can be recognised; we follow Bloembergen (1939) and Hewson (1984) in ranking them as subspecies.

\section{Key to the subspecies}

1. Indument composed of hairs $0.1-0.5 \mathrm{~mm}$ long, plant early glabrescent. Internodes glabrous a. subsp. polyosmoides

1. Indument of hairs (0.3-) $0.5 \mathrm{~mm}$ long, persistent. Internodes hairy . . . . . . . . . . . . . bubsp. tomentosum

\section{a. subsp. polyosmoides}

Alangium villosum (Blume) Wangerin subsp. polyosmoides (F.Muell.) Bloemb. (1939) 209, f. 6j, 7t-u; Hewson (1984) 11, f. 3, map 13.

Twigs glabrous or glabrescent. Lamina glabrous, (narrowly) elliptic, base asymmetric, long-cuneate. Flowers short-hairy, corolla in bud 8-15 mm long; style sparingly hairy.

Distribution - Australia, Queensland (Cape York Peninsula) to New South Wales.

Habitat \& Ecology — Rain forest.

Note - The collection Ford 04301 (QRS n.v.; L) from Hutton Drive, Tonga, Claudie River area, cultivated, germinated from Starling poo, has exceedingly small flowers, the corolla in bud estimated c. $8 \mathrm{~mm}$ long. 

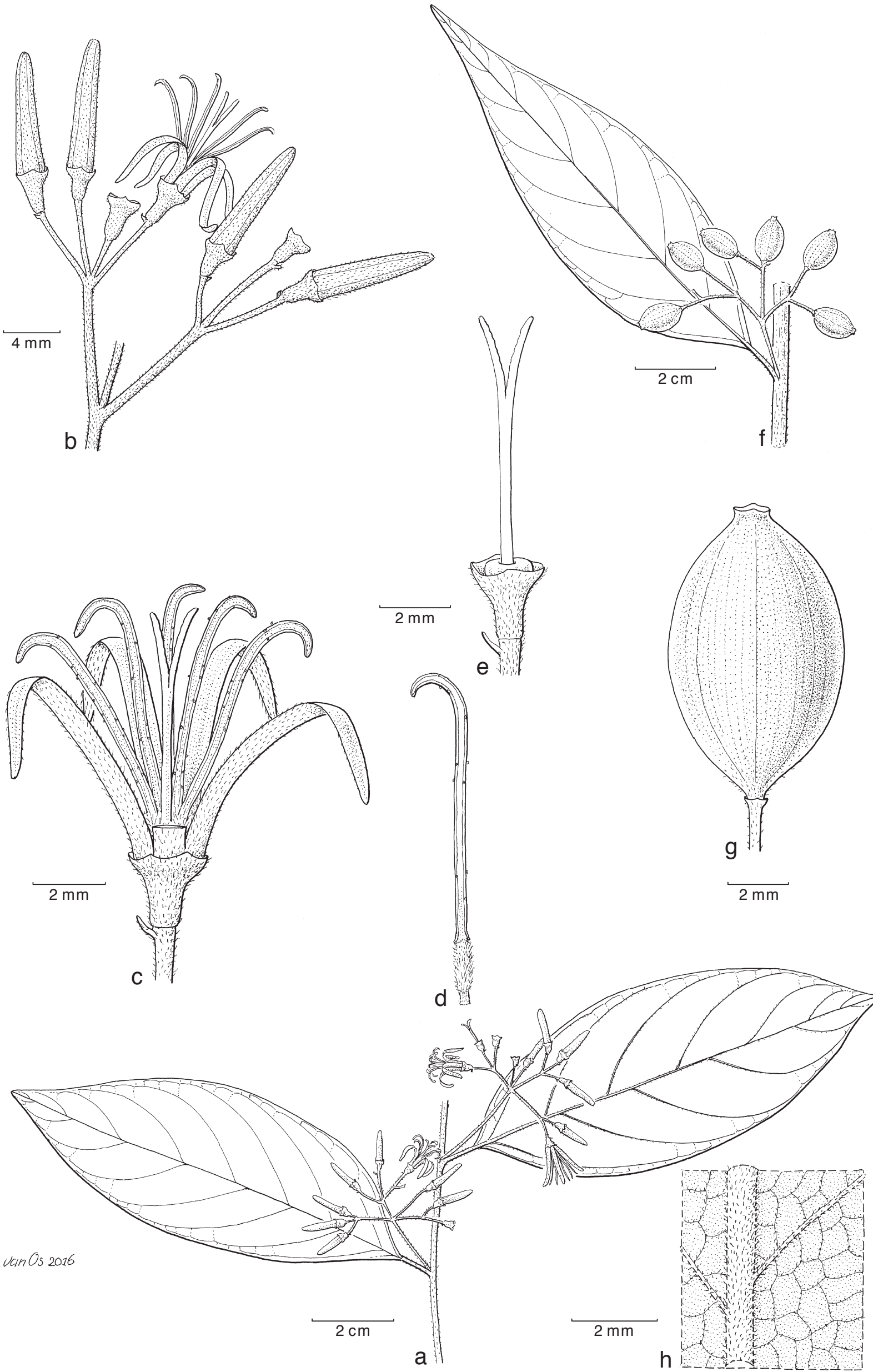

Fig. 2 Alangium pilosum Merr. a. Inflorescences; b. apical part of inflorescence; c. flower; d. stamen; e. ovary, disc and style (petals removed), (note stigma deeply 2-lobed); f. infructescence; g. fruit; h. lower lamina surface (a-e, h: Verheijen 1843 (Flores); f, g: Kostermans \& Wirawan 122 (Flores); all L). — Drawn by Jan van Os. 
b. subsp. tomentosum (F.Muell.) W.J.de Wilde \& Duyfjes, comb. nov.

Pseudalangium polyosmoides (F.Muell.) Baill. var. tomentosum ('tomentosam') F.Muell. (1860) 85. - Marlea vitiense (A.Gray) Benth. var. tomentosa (F.Muell.) Benth. (1867) 386. - Alangium vitiense (A.Gray) Benth. var. tomentosum (F.Muell.) Wangerin (1906) 63. - Alangium villosum (Blume) Wangerin var. tomentosum (F.Muell.) Bloemb. (1935) 271, p.p. [for the type only]. - Alangium villosum (Blume) Wangerin subsp. tomentosum (F.Muell.) Bloemb. (1939) 203, f. 6b-b'; Hewson (1984) 13, map 14. - Alangium villosum (Blume) Wangerin subsp. tomentosum (F.Muell.) Bloemb. var. australe Bloemb. (1939) 204, f. 6b', 7g, nom. illeg. (cited material includes the type of var. tomentosum F.Muell.) - Lectotype here designated: Hill s.n. (K K000704818; iso MEL n.v.), Australia, Brisbane River.

Twigs conspicuously hairy, hairs c. $0.5 \mathrm{~mm}$ long. Lamina hairy, (broadly) elliptic or ovate-elliptic, base strongly asymmetric, broadly rounded to short-cuneate. Flowers conspicuously hairy, corolla in bud 8-14 mm long; style densely hairy.

Distribution - Australia, Queensland (Atherton Tableland) to north coast of New South Wales.

Habitat \& Ecology - Rain forest.

Note - The specimen McDonald \& Stainton 2305 (BRI n.v., $\mathrm{L})$, Queensland, from andesite base rock, has exceedingly small flowers, corolla in bud estimated as c. $6 \mathrm{~mm}$ long only. Apparently in both subspecies the flower size is quite variable.

\section{Alangium solomonense (Bloemb.) W.J.de Wilde \& Duyfjes,} comb. \& stat. nov.

Alangium villosum (Blume) Wangerin subsp. solomonense Bloemb. (1939) 207, f. 6g, 7o-p. - Lectotype here designated: Brass 3463 (BO; iso An.v., BISH BISH1000643, L 2 sheets L.2500050, L.2500051), Solomon Islands, Santa Ysabel Isl., Sigana, 12 Jan. 1933, fl., fr.

Tree 7-17 m tall; twigs blackish brown, 1.5-3 mm diam, (greyish) brown-hairy in the youngest part, hairs c. $0.1 \mathrm{~mm}$ long. Leaves: petiole (0.5-) $0.8-1 \mathrm{~cm}$ long, hairy as the twigs; lamina drying greenish brown, early glabrescent from minute hairs less than $0.1 \mathrm{~mm}$ long, (ovate-)elliptic, $6-13$ by $2-5 \mathrm{~cm}$, base asymmetric, cuneate, apex acute-acuminate; veins pinnate, 4-6 at each side, tertiary venation thin, reticulate. Inflorescences minutely hairy (hairs \pm sparse, less than $0.1 \mathrm{~mm}$ long), a single long peduncled compound cyme, 5-15-flowered; peduncle 5-12 mm long. Flowers densely pale brown-hairy, hairs c. 0.1 $\mathrm{mm}$ long; pedicel 3-7 mm long; corolla in bud (5-)6-8 mm long, base not swollen, apex (sub)obtuse; ovary and calyx (1.5-) $2 \mathrm{~mm}$ long, not ribbed; limb narrowly cup-shaped, spreading, c. $0.5 \mathrm{~mm}$ long, c. $1 \mathrm{~mm}$ wide at margin, lobes 5 (or 6), c. 0.5 $\mathrm{mm}$ long; petals 5 or 6 , inside glabrous, $7-8 \mathrm{~mm}$ long, at base (less than) $1 \mathrm{~mm}$ connate; stamens 5 (or 6), filament c. $2.5 \mathrm{~mm}$ long, hairy, anther 4-5 mm long, connective glabrous; style 4-6 $\mathrm{mm}$ long, hairy, stigma lobes $1-1.5 \mathrm{~mm}$ long. Fruit (1-)2-5 per infructescence, ripening yellowish green?, glabrescent, ovoidellipsoid, much narrowed towards apex, 17-22 mm long, faintly narrowly ribbed; calyx remnant narrow, small.

Distribution - Solomon Isl., widespread; in Malesia: Papua New Guinea, Bougainville, where only known from Volk \& Robinson NGF 590 (L).

Habitat \& Ecology - Well drained primary rain forest, on flat plain, ridge side and ridge top; brown sandy loam and coral; from sea level to $300 \mathrm{~m}$ altitude; flowering and fruiting all year round.

Field-notes - Common in lowland rain forest; buttresses mostly absent.

Notes - 1. Alangium solomonense resembles A. pilosum, the latter differs in a slightly smaller corolla, a glabrous style, and smaller fruit.

2. The fruiting pedicel of $A$. solomonense is $5-10(-20) \mathrm{mm}$ long, longer than generally in the other species of the section.

\section{Alangium strigosum W.J.de Wilde \& Duyfjes, nom. nov.}

lodes ferruginea Lauterb. \& K.Schum. in Schumann \& Lauterbach (1900) 418 (see note 1). - Type: Lauterbach 2387 (see note 2) (holo B十, lectotype here designated: WRSL; iso G-BOISS n.v., K K000704816, L L0009827), Papua New Guinea, Kaiser Wilhelmsland, Schumann Fluss, Lager II, 250 m alt., 20 June 1896, fr.

Tree c. $10 \mathrm{~m}$ tall; twigs brown, 2-3.5(-6) mm diam, densely rusty or golden brown-hairy, hairs $0.5-1 \mathrm{~mm}$ long. Leaves: petiole $0.5-1 \mathrm{~cm}$ long, hairy; lamina drying greenish, soft pale hairy below (glabrescent above), hairs $0.1-0.5 \mathrm{~mm}$ long, elliptic, 13-19 by $4-9 \mathrm{~cm}$, base asymmetric, cuneate, apex acute-acuminate; veins pinnate, $4-5(-7)$ at each side, tertiary venation widereticulate. Inflorescences hairy (hairs less than $0.5 \mathrm{~mm}$ long), simple, 5-10-flowered, peduncle 8-10 mm long. Flowers (only immature buds known) densely golden or greyish brown-hairy; pedicel c. $2 \mathrm{~mm}$ long; corolla in bud presumably c. $10 \mathrm{~mm}$ long, c. $1.5 \mathrm{~mm}$ diam, base not swollen, apex obtuse; ovary and calyx limb c. $4 \mathrm{~mm}$ long, not ribbed, yellowish or greyish brown-hairy, limb c. $1 \mathrm{~mm}$ long, with lobes nearly $1 \mathrm{~mm}$ long, not spreading; petals 5, inside glabrous; stamens 5 , filament c. $2 \mathrm{~mm}$ long, thinly hairy, anther c. $3 \mathrm{~mm}$ long, connective glabrous; style glabrous but hairy towards and on stigma lobes. Fruit c. 2 per infructescence, ripening red, glabrescent, ellipsoid, 12-15 by c. $10 \mathrm{~mm}$, not ribbed, apex not narrowed; calyx remnant small, narrow, consisting of 4 (or 5 ) strigose connivent lobes C. $1 \mathrm{~mm}$ long.

Distribution - Papua New Guinea (Madang and Sepik Provinces).

Habitat \& Ecology - Rain forest, low hilly terrain; clay and marl; 50-250 m altitude; flowering in October; fruiting in June.

Notes - 1. Alangium strigosum needed a new name, because it was previously described as lodes ferruginea (in Icacinaceae) which cannot be combined in Alangium because of $A$. ferrugineum.

2. The lectotype, in WRSL, bears the number Lauterbach 387 , but in the protologue the number is changed into Lauterbach 2387 , presumably the number 2 is added to indicate that the collection was from 'Lager' = camp 2 .

3. Alangium strigosum resembles $A$. velutinum, the latter is a montane species distinct in conspicuous dense red-brown indument.

4. The species is known from two collections: the type and Pullen 1810 (L), from Sepik Prov., Wewak-Angoram Area, c. 50 m alt., 1 Oct. 1959, fl.

\section{Alangium velutinum W.J.de Wilde \& Duyfjes, sp. nov.}

Resembling Alangium ferrugineum C.T.White but differing in its velutinous surface of the lamina below, and c. $15 \mathrm{~mm}$ long fruit with acute apex (lamina less densely hairy below, fruit c. $35 \mathrm{~mm}$ long and longly narrowed towards apex in A. ferrugineum). - Type: Grubb \& Edwards 79 (holo L L2500083; iso CANB n.v.), Papua New Guinea, Eastern Highlands Province, W of Fatima River, Marafunga Mill, Goroka Sub-Dist., S605' E145¹5', 2600 m alt., 30 Nov. 1970, fl., fr.

Etymology. The specific epithet 'velutinum' refers to the velvety surface of the lamina below.

Tree 5-13 m tall; twigs brown, 2-4 mm diam, reddish brown or rusty brown-hairy, hairs $0.3-0.5 \mathrm{~mm}$ long. Leaves: petiole $0.6-1 \mathrm{~cm}$ long, hairy; lamina drying reddish brown, glabrescent above, velutinous below all over, hairs c. $0.5 \mathrm{~mm}$ long, (obovate-)elliptic, $6-15$ by $2.5-7 \mathrm{~cm}$, base distinctly or indistinctly asymmetric, cuneate, apex acute-acuminate; veins pinnate, 4 or 5 at each side, tertiary venation reticulate-scalariform. Inflorescences hairy as the twigs, simple, 4-10-flowered; peduncle 5-10 mm long, 1-2 mm diam. Flowers densely hairy; pedicel 2-5 mm long; corolla in bud (fully mature bud not seen) 9-10 $\mathrm{mm}$ long, c. $1.5 \mathrm{~mm}$ diam, base not swollen, apex obtuse; ovary 
and calyx 3-4.5 mm long, not ribbed, reddish brown-hairy; limb c. $1 \mathrm{~mm}$ long, not spreading, with subacute lobes $0.5-1$ $\mathrm{mm}$ long; petals 5 , inside glabrous but somewhat hairy at very base, c. $9 \mathrm{~mm}$ long, connate at base into a tube c. $3 \mathrm{~mm}$ long; stamens 5, filament c. $4 \mathrm{~mm}$ long, hairy, anther c. $5 \mathrm{~mm}$ long, connective glabrous; style c. $4 \mathrm{~mm}$ long, glabrous, stigma lobes 1.5-2 mm long. Fruit 2-6 per infructescence, ripening red, glabrescent, ellipsoid, c. $15 \mathrm{~mm}$ long, apex acute, not narrowed; calyx remnant narrow, not spreading.

Distribution - Papua New Guinea (Eastern Highlands Prov., and Morobe Prov. (doubtful)).

Habitat \& Ecology - Primary montane forest, low forest on creek bank; 1400-2600 m altitude; flowering in June and November; fruiting in April, September and November.

Field-notes - Leaves redly tomentose on reverse; flowers cream or white; fruit red; seed dark greyish brown with whitish spots; Womersley \& Sleumer NGF 14006: 'overall in mountain forest'.

Vernacular name - Monr (Marfunga Mill).

Additional specimens studied. PAPUA New GuINEA, Eastern Highlands Prov., Goroka Subdistrict, W of Fatima River, Marafunga Mill: Grubb \& Edwards 11, S6 ${ }^{\circ} 5^{\prime} \mathrm{E} 145^{\circ} 15^{\prime}, 2600$ m alt., 10 Nov. 1970, fl.; Grubb \& Edwards 193, S6 ${ }^{\circ} 05^{\prime} \mathrm{E} 145^{\circ} 11^{\prime}, 2600 \mathrm{~m}$ alt., 26 Apr. 1971, fr.; Millar NGF 40781, slopes of Kerigomna, $\mathrm{S}^{\circ}{ }^{\circ} 5^{\prime} \mathrm{E} 145^{\circ} 15^{\prime}, 2450 \mathrm{~m}$ alt., 14 Nov. 1968, fl.; Womersley \& Sleumer NGF 14006, Marafunga logging area, upper Asaro Valley, S6 ${ }^{\circ} 05^{\prime}$ E145¹5', 2450 m alt., Sept. 1961, fl. Morobe Prov.: Hoogland 9183, E slope of Mt Rawlinson, Huon Peninsula, c. 1400 m alt., 16 June 1964 (somewhat doubtful, immature buds only).

Note - Alangium velutinum also resembles $A$. strigosum, the latter differs in the lamina which are green on drying and much less conspicuous soft hairy below.

\section{Alangium villosum (Blume) Wangerin}

Alangium villosum (Blume) Wangerin (1906) 61; Bloemb. (1935) 269, f. 3a, f; (1939) 198. - Marlea villosum (Blume) Kurz (1871) 61. - Styrax villosum Blume (1826) 671. — Lectotype here designated: Blume s.n. (L L0009830; iso L 3 sheets L0614166, L0614168, L0614169), Java.

Alangium villosum (Blume) Wangerin var. salaccense (Koord. \& Valeton) Bloemb. (1935) 271, nom. illeg. - Alangium villosum (Blume) Wangerin subsp. salaccense (Koord. \& Valeton) Bloemb. (1939) 202, nom. illeg. - Marlea vitiensis (A.Gray) Benth. var. tomentosa (F.Muell.) Benth. forma salaccensis Koord. \& Valeton (1900) 75, nom. illeg.

Alangium villosum (Blume) Wangerin subsp. tomentosum (F.Muell.) Bloemb var. javanum Bloemb. (1939) 204, f. 6b, 7a, f. - Lectotype here designated: Koorders 38191 (L L.2500069; iso BO n.v.), Java, Pasoeroean, MtArdjoeno, 1400 m alt., 10 Nov. 1899, fl.

Alangium zollingeri Baill. (1864) 195. - Type: Zollinger $785 Z$ (holo P P04552612), Java.

Alangium villosum (Blume) Wangerin var. tomentosum auct. non (F.Muell.) Bloemb.: Bloemb. (1935) 271, f. 3a, f.

Tree presumably of low stature (but Backer (1965) says 10-20 m tall); twigs (1.5-)2-4 mm diam, (greyish) brown-hairy, hairs $0.2-0.5(-1) \mathrm{mm}$ long. Leaves: petiole $0.3-1(-1.2) \mathrm{cm}$ long, hairy; lamina drying brown, hairy like the twigs, glabrescent above, ovate-elliptic or (narrowly) elliptic, $6-14(-19)$ by $2-5(-7)$ $\mathrm{cm}$, base little or strongly asymmetric, rounded or short- or long-cuneate, apex acute-acuminate; veins pinnate, 5 or 6 at each side, tertiary venation reticulate, sometimes \pm scalariform, domatia absent. Inflorescences solitary peduncled simple or compound cymes, hairy as the twigs, (2-)5-30-flowered; peduncle (3-)5-15 mm long. Flowers densely hairy like the twigs; pedicel 2-4 mm long; corolla in bud (6-)7-8 mm long, base not or little swollen, apex (sub)obtuse; ovary and calyx c. $2 \mathrm{~mm}$ long, not ribbed; limb c. $0.5 \mathrm{~mm}$ long, at margin c. 2 $\mathrm{mm}$ wide, with 5 low triangular lobes c. $0.5 \mathrm{~mm}$ long; petals $(4-) 5(-7)$, inside (sparingly or towards base strongly) hairy, not connate at base, c. $8 \mathrm{~mm}$ long; stamens 5 , not adnate to petals, filament c. $3 \mathrm{~mm}$ long, hairy, anthers c. $4 \mathrm{~mm}$ long, connective glabrous; style 5-6 mm long, glabrous, stigma lobes c. $1 \mathrm{~mm}$ long. Fruit 2-7 per infructescence, ripening dark red (Backer 1965), glabrescent, ovoid or broadly ellipsoid, (8-)10-15 mm long, smooth or inconspicuously ribbed; calyx remnant minute.

Distribution - West and East Java.

Habitat \& Ecology - Montane and lower montane forest; (from lowland to) 700-1800 m altitude; flowering from August to December.

Notes - 1. Specimens form West Java (incl. the type) are rare and scanty and apparently more slender of stature (leaves narrower, inflorescences poorer flowered) as compared with those from East Java. Recent collections are not known.

2. According to Koorders \& Valeton (1900) the bark and leaves are very bitter.

\section{Alangium vitiense (A.Gray) Baill.}

Alangium vitiense (A.Gray) Baill. (1864) 195. - Alangium villosum (Blume) Wangerin subsp. vitiense (A.Gray) Bloemb. (1939) 208, f. 6i, 7r-s. - Rhytidandra vitiensis A.Gray (1854) 303, t. 28. - Lectotype here designated: Unknown s.n. (US US 00127567; iso US US 00127568), Fiji Isl. ('Feejee'). Alangium bussyanum (Baill.) Harms (1898) 262. - Alangium villosum (Blume) Wangerin subsp. bussyanum (Baill.) Bloemb. (1939) 207, f. 6h, 7q. - Marlea bussyana Baill. (1872) 183. — Type: Pancher s.n. (holo P P00522317), New Caledonia fl.

(Shrub or) tree, 3-20 m tall; twigs usually blackish, sometimes conspicuously lenticellate, (1-)2-4 mm diam, glabrous (early glabrescent from grey appressed hairs $0.1 \mathrm{~mm}$ long or less). Leaves: petiole (0.5-)1-1.5 cm long, glabrous; lamina drying blackish or brown, membranous or subcoriaceous, ovateelliptic, $8-16$ by $3.5-9 \mathrm{~cm}$, base strongly asymmetric, (broadly) rounded or cuneate, apex acute-acuminate; veins pinnate, 5-7 on each side, tertiary venation finely or coarsely (sub)scalariform. Inflorescences glabrous, solitary peduncled compound cymes, (2-)5-10-flowered; peduncle 5-25(-40) mm long. Flowers densely minutely grey-hairy; pedicel 2-7 mm long; corolla in bud 7-8 mm long, base not swollen, apex (sub)obtuse; ovary and calyx 2-3.5 mm long, not ribbed; limb spreading, 1-1.5 mm long, 2-3(-4) mm wide at margin, shallowly lobed, lobes c. $0.5 \mathrm{~mm}$ long; petals 4-6, inside glabrous or sparingly hairy, 7-9 mm long, connate at base for c. $1 \mathrm{~mm}$; stamens 5 , at base slightly adnate to petals, $8-10 \mathrm{~mm}$ long, filament 3-4 $\mathrm{mm}$ long, hairy in the middle, anthers $5-6 \mathrm{~mm}$ long, connective glabrous; style 5-7 mm long, glabrous or sparingly hairy (New Caledonia), stigma lobes c. $1.5 \mathrm{~mm}$ long. Fruit (1-)2-7 per infructescence, ripening purple to black, glabrous, ovoid-ellipsoid, 17-22 by $8-11 \mathrm{~mm}$, smooth; calyx remnant cup-shaped, \pm spreading, c. $2 \mathrm{~mm}$ wide at apex.

Distribution - Fiji Isl., Vanuatu, New Caledonia.

Habitat \& Ecology — Lowland rain forest, scrub-land, coralline terrace (New Caledonia); brown-red soil; 100-700 m altitude; flowering from March to August; fruiting from March to December.

Field-notes - Chew Wee-Lek RSNM 60: 'Very common as 'poles' in Kauri forest' (Vanuatu); fruit becoming purple to black.

Note - Specimens from New Caledonia generally have been collected from shrubs and have smaller, somewhat less unequal laminas and shorter peduncles ( $5 \mathrm{~mm}$ long) as compared with specimens from Fiji and Vanuatu. In New Caledonia the flowers are comparatively small (corolla c. $7 \mathrm{~mm}$ long) and, once seen in Guillaumin et al. 11832, the petals inside and the style are sparingly hairy. 


\section{Alangium warburgianum Wangerin}

Alangium warburgianum Wangerin (1910) 18. - Alangium villosum subsp. warburgianum (Wangerin) Bloemb. (1935) 273, f. 3i-I; (1939) 205, f. 6e, 7j-I. - Type: Warburg 18116 (holo B十, lectotype here designated: A, A00054249), Moluccas, Bacan Isl., Sibella, 750-1200 m altitude.

Shrub or treelet, 1.5-5 m high; twigs dark brown, 1.5-3 mm diam, glabrescent, at very apex with dense appressed rusty hairs (0.1-) $0.2 \mathrm{~mm}$ long; leaf bud densely rusty hairy. Leaves: petiole $0.7-1.5 \mathrm{~cm}$ long, hairy like twig apex; lamina drying brown, early glabrescent, (narrowly) elliptic, $9-16$ by 3-4 $\mathrm{cm}$, base slightly asymmetric, cuneate, apex (long) acuteacuminate; veins pinnate, $5-7$ on each side, tertiary venation reticulate. Inflorescences sub-persistently hairy like twig apex, simple, 2-5-flowered, peduncle 5-10 mm long. Flowers densely rufous-hairy, hairs $0.2-0.3 \mathrm{~mm}$ long; pedicel $2-3 \mathrm{~mm}$ long; corolla in bud 9-10 mm long, base not swollen, apex narrow, subobtuse; ovary and calyx densely hairy, c. $2.5 \mathrm{~mm}$ long, not ribbed; limb $1.5 \mathrm{~mm}$ long, mainly consisting of 5 long-triangular lobes c. $1.5 \mathrm{~mm}$ long, not spreading; petals (4 or) 5 , inside glabrous, 8-10 mm long; stamens 5, c. $9 \mathrm{~mm}$ long, filament 2-3 mm long, hairy inside, anther 5-6 mm long, connective glabrous; style 5-6 mm long, (sparingly) hairy, stigma lobes c. $2 \mathrm{~mm}$ long. Fruit 1-3 per infructescence, ripening red, glabrescent, ovoid-ellipsoid or ellipsoid, narrowed at base and apex (longest at apex), 15-22 mm long, finely 5-ribbed or smooth; calyx remnant minute, the lobes \pm connivent.

Distribution - Moluccas (endemic to Bacan Isl.).

Habitat \& Ecology - Dense primary forest 20-35 m high, hill ridge; clayey soil, bedrock grey schists; $900-1250$ m altitude; flowering and fruiting in October.

Field-notes - Fruit red, whitish hyaline pulp.

Notes - 1. Alangium warburgianum is known from four collections, all from Bacan Isl., Gunung Sibela: Boschproefstation b.b. 23243 (BO), $1000 \mathrm{~m}$ alt.; De Vogel 3583 (L), $1250 \mathrm{~m}$ alt., 23 Oct. 1974, fl. \& fr.; De Vogel 3644 (L), 900 m alt., 24 Oct. 1974, fr.; De Vogel 3662 (L), 1000 m alt., 25 Oct. 1974, fl. \& fr.

2. The peduncle is remarkably slender, comparable with the slender peduncle of $A$. gracile and A. pilosum.

\section{DOUBTFUL COLLECTIONS}

Five mutually different collections (all in L), one with fruit and 4 with flowers, obviously belong to the group of species around A. ferrugineum, but we cannot satisfactorily link them up with any of the accepted species. They cannot be described adequately as new species either by lack of flowers or of fruit, and one should wait for additional materials to be collected. The three first listed possibly belong closer together and are near $A$. glabrum.

The five specimens now labelled as Alangium sp. sect. Rhytidandra are the following:

PAPUA, Van Royen \& Sleumer 8126, Vogelkop Peninsula, Nettoti range, $1550 \mathrm{~m}$ alt., 4 Dec. 1961, fl. (distinct in hairy filaments, which are for over halfway adnate to the tubular connate base of the petals). - PAPUA New Guinea, Morobe Prov.: Craven \& Schodde 1470, near Wengia, Aseki Patrol Area, 1100 m alt., 28 Apr. 1966, fl.; Kairo 779, Gumi Divide, 25 km W of Bulolo, S7 ${ }^{\circ} 13^{\prime} \mathrm{E} 146^{\circ} 25^{\prime}, 1800 \mathrm{~m}$ alt., fl.; Central Prov.: Carr 14510, Boridi, 1370 m alt., 10 Nov. 1935, fl.; Northern Prov.: James et al. 1075, Sibium Mts, S9³0' E148²9', 1315 m alt., fl., fr.
Acknowledgements The materials necessary for the present study were basically those of herbarium collections in $L$ (including $U$ ) of which most older collections were identified by the last monographer Bloembergen. In addition we thank the curators in BO, K, and SING for allowing us to study their collections and we thank Krzysztof Świerkosz (WRSL) for providing a photo of the holotype of lodes ferruginea and Danielle Hanharan (A) for a photo of the isotype of $A$. warburgianum. The study in SING was made possible by a grant generously provided by the Singapore herbarium. The illustrations are as usual by the Leiden artist Jan van Os.

\section{REFERENCES}

Backer CA. 1965. Alangiaceae. In: Backer CA, Bakhuizen van den Brink RC, Flora of Java 2: 159-161. Noordhoff, Groningen.

Baillon H. 1864-1865. Recherches sur l'Aucuba et sur ses rapports avec les genres analogues. Adansonia; recueil (périodique) d'observations botaniques, Paris 5: 179-203. Paris.

Baillon H. 1872. Stirpes Exoticae Novae. Adansonia; recueil (périodique) d'observations botaniques 10: 183. Paris.

Baillon H. 1876. Histoire des plantes 6: 270, 276. Librairie Hachette \& Cie, Paris.

Bentham G. 1867. Flora Australiensis 3: 386. Reeve \& Co., London.

Bloembergen S. 1935. The genus Alangium in the Netherlands Indies. Blumea 1: 241-294.

Bloembergen S. 1939. A revision of the genus Alangium. Bulletin du Jardin Botanique Buitenzorg, série 3, 16: 139-235.

Blume CL. 1826 '1825'. Bijdragen tot de flora van Nederlandsch Indië 13: 671. Ter Lands Drukkerij, Batavia.

De Wilde WJJO \& Duyfjes BEE. 2017. Taxonomy of Alangium section Conostigma (Alangiaceae). Blumea 62: 29-46.

De Wilde WJJO \& Duyfjes BEE. In press. Conspectus of Alangium sect. Alangium (Alangiaceae). Thai Forest Bulletin (Botany).

Gray A. 1854. United States Exploring Expedition. During the years 1838 1839, 1840, 1841, 1842. Under the Command of Charles Wilkes, U.S.N. vol. XV: 302, 303. Botany. Phanerogamia by Asa Gray with a Folia Atlas of 100 Plates. Part 1. Philadelphia.

Harms H. 1898. Cornaceae. In: Engler HGA (ed), Die natürlichen Pflanzenfamilien 3: 259-270. Engelmann, Leipzig.

Hewson HJ. 1984. Alangiaceae. Flora of Australia 22: 11-13. Australian Government Publishing Service, Canberra.

Koorders SH, Valeton Th. 1900. Cornaceae. Bijdrage no. 5 tot de kennis der boomsoorten op Java. Mededelingen uit's Lands Plantentuin 33: 65-101. Kolff \& Co. Batavia.

Kurz S. 1871. Cornaceae. Journal of the Asiatic Society of Bengal, Part 2, Natural History 40: 61.

Merrill ED. 1922. Alangiaceae. Philippine Journal of Science 20: 417, 418.

Merrill ED. 1923. Alangiaceae. An enumeration of Philippine flowering plants 3: 240-241. Bureau of Printing, Manilla.

Schumann K, Lauterbach K. 1900. Die Flora der Deutschen Schutzgebiete in der Südsee: 418. Bornträger, Leipzig.

Von Mueller FJH. 1860. Alangiaceae. Fragmenta Phytographiae Australiae 2: 84-85. Melbourne.

Von Mueller FJH. 1864. Additamenta ad volumen secundum. Fragmenta Phytographiae Australiae 4: 172. Melbourne.

Wangerin W. 1906. Die Gattung Alangium. Botanische Jahrbücher für Systematik, Pflanzengeschichte und Pflanzengeographie, Beiblatt 86: 61-88. Wangerin W. 1910. Alangiaceae. In: Engler HGA, Das Pflanzenreich 41 IV.220b: 1-24. Engelmann, Leipzig.

White CT. 1929. Alangiaceae. Journal of the Arnold Arboretum 10: 248-249. 